\title{
A relação das Habilidades Sociais educativas e das práticas educativas maternas com os problemas de comportamento em adolescentes ${ }^{1}$
}

\section{The relation between social educative skills and maternal educative practices with adolescent behavior problems}

\section{Gabriela Mello Sabbag*}

Faculdade de Administração, Ciências, Educação e Letras -FACEL, Curitiba, PR, Brasil

\author{
Alessandra Turini Bolsoni-Silva** \\ Universidade do Estado de São Paulo - UNESP, SP, Brasil
}

\begin{abstract}
RESUMO
Este estudo buscou correlacionar as habilidades sociais educativas e as práticas educativas maternas com as habilidades sociais e os problemas de comportamento dos adolescentes do sexo feminino e masculino, considerando os grupos de risco e os de não risco para problemas de comportamento. Para tanto, foram investigados os comportamentos maternos através do Roteiro de Habilidades Sociais Educativas Parentais (RE-HSE-P) e do Inventário de Estilo Parental (IEP). Os comportamentos dos adolescentes foram avaliados através do RE-HSE-P e do Child Behavior Checklist. Participaram do estudo 24 mães e seus filhos adolescentes dos grupos de risco e de não risco. Os resultados apontaram para correlações positivas entre as habilidades sociais educativas maternas no RE-HSE-P e as práticas educativas maternas no IEP. As comparações entre os grupos de risco e os de não risco mostraram que as mães dos adolescentes do grupo de risco apresentaram maior frequência de práticas negativas. Seus filhos mostraram menor frequência de habilidades sociais e maior frequência de problemas de comportamento. Não foram encontradas divergências entre as práticas maternas aplicadas aos adolescentes do sexo masculino e feminino. Também não houve diferenças quanto às habilidades sociais e aos problemas de comportamento em meninos e meninas.
\end{abstract}

Palavras-chave: Habilidades sociais educativas maternas, Adolescentes, Problemas de comportamento.

\begin{abstract}
This study correlated maternal social educative skills, negative maternal practices and social skills and behavior problems in male and female adolescents groups of risk and not risk for behavior problems. The maternal behavior was investigated by Parental Educative Social Skills Script Interview (RE-HSE-P) and by Parental Styles Inventory (IEP). The adolescent behavior had been evaluated through the RE-HSE-P and of the Child Behavior Checklist. 24 mothers and adolescents of the groups of risk and not risk had participated of the study. The results had pointed to positive correlations between the maternal social educative skills in the REHSE-P and maternal practices in the IEP. The comparisons between the groups of risk and not risk showed that the mother's risk group had
\end{abstract}


presented more often negative practices and it's teenagers had showed less social skills and more behavior problems. Divergences between maternal practices applied to boys and girls were not found. Also it did not have differences about the social skills and the behavior problems in boys and girls.

Keywords: Maternal educative social skills, Adolescents, Behavior problems.

A literatura especializada no relacionamento entre pais e filhos adolescentes associa as práticas parentais ao desenvolvimento de habilidades sociais ou de problemas de comportamento (AVANCI, et al., 2007; PACHECO; SILVEIRA; SCHNEIDER, 2008; REICHERT; WAGNER, 2007; TEIXEIRA; OLIVEIRA; WOTTRICH, 2006; FLETCHER; STEINBERG; WILLIAMS-WHEELER, 2004; PATTERSON; REID; DISHION, 2002; STATTIN; KERR, 2000).

Diversos modelos teóricos mostram que as práticas parentais de risco são vinculadas a uma cadeia de causas que inicia com comportamentos desviantes na infância, podendo levar à associação de pares desviantes e resultando no comportamento antissocial na adolescência (PATTERSON; REID; DISHION, 2002; GOMIDE, 2003, 2004, 2006; LONG; FOREHAND, 2003).

Pesce, Assis, Santos e Oliveira (2004), preocupados em identificar variáveis correlacionáveis com o constructo de resiliência junto a 997 adolescentes, verificaram que a violência física, a psicológica (consideradas práticas negativas de educação por Gomide, 2003) e outros itens de violência não correlacionaram. Ao contrário, o apoio social, a supervisão familiar e o relacionamento com amigos e professores foram associados à resiliência. Sendo assim, a supervisão parental e o apoio parecem promover o bem-estar e o ajustamento do adolescente, ao contrário das práticas negativas de educação.

Outras pesquisas também atestam para a importância da supervisão ou monitoria parental na prevenção de problemas de comportamento na adolescência, bem como para a presença de monitoria negativa associada à ansiedade e depressão (SALVO; SILVARES; TONI, 2005; BORAWSKI et al., 2003; DICLEMENTE et al., 2001; FLETCHER; STEINBERG; WILLIAMS-WHEELER，2004; GOMIDE，2004，2006; STATTIN; KERR, 2000). Além do risco para problemas de comportamento, as práticas educativas negativas também aparecem associadas a indicadores de abuso de substâncias na adolescência (FLETCHER; STEINBERG; WILLIAMS-WHEELER, 2004; SCHENKER; MINAYO, 2005).

O contrário das práticas educativas negativas são as denominadas práticas educativas positivas (GOMIDE, 2006). Estas foram classificadas em monitoria positiva e comportamento moral, e são práticas estimuladoras de comportamentos pró-sociais dos filhos. Aquelas que possibilitam a ocorrência de problemas de 
comportamento foram denominadas de práticas negativas, tais com: disciplina relaxada, punição inconsistente, monitoria negativa, abuso físico e negligência (GOMIDE, 2006).

Borawski et al. (2003) alertam, no entanto, que a maioria das pesquisas tem se voltado para o estudo da dimensão monitoria parental, como citado anteriomente. Borawski et al. (2003) perceberam que a monitoria parental, definida como saber onde e com quem o filho está, quando utilizada sem outros comportamentos por parte dos pais, não é suficiente para a prevenção dos comportamentos de risco. Na mesma direção, no que diz respeito ao comportamento de negociação e orientação da conduta do adolescente, Stattin e Kerr (2000) enfatizam a importância da comunicação positiva entre pais e filhos para que a monitoria seja efetiva, isto é, seja o principal instrumento de prevenção contra problemas de comportamento.

Tendo em vista que as práticas positivas e negativas dos pais influenciam o comportamento dos filhos e que a literatura, que estuda o comportamento de ambos conjuntamente, é escassa, considera-se relevante ampliar o conjunto de comportamentos denominados práticas positivas. É relevante também descrever o comportamento dos pais em contingência ao comportamento dos filhos.

Com essa preocupação, Bolsoni-Silva $(2008,2009)$ busca realizar a análise funcional dos comportamentos dos pais e dos filhos. A autora propõe a análise de três termos (SKINNER, 1998) aplicada à relação entre pais e filhos. Nessa análise são investigados os estímulos antecedentes às ações parentais e filiais, as respostas comportamentais dos pais e dos filhos, assim como as consequências advindas dessas interações.

Dentro desse contexto de análise, busca-se a identificação das habilidades sociais educativas parentais, das práticas negativas parentais, das situações de contexto interativo e dos comportamentos do filho, os quais podem ser considerados socialmente habilidosos ou problemáticos. Entretanto, a pesquisadora teve como foco crianças pré-escolares, o que justifica a ampliação do estudo para a adolescência. Com a ampliação, pode-se verificar se há ou não padrões que se apresentam similares ou diferentes dos manifestados na infância.

As habilidades sociais são comportamentos utilizados em interações sociais que trazem benefícios para o indivíduo e para as demais pessoas (DEL PRETTE; DEL PRETTE, 1999). Para a categorização das habilidades sociais educativas parentais em três grandes áreas, comunicação, expressividade e estabelecimento de limites, BolsoniSilva (2008) baseou-se em autores da área de habilidades sociais (DEL PRETTE; DEL PRETTE, 1999, CABALLO, 2002), pesquisadores envolvidos no estudo de práticas parentais (por exemplo, 
PATTERSON; REID; DISHION, 2002; WEBSTER-STRATTON; REID; HAMMOND, 2003) e consultou autores da Análise do Comportamento, especialmente quanto à utilização da análise funcional em prática clínica (por exemplo, MEYER et al., 2008).

Em relação à qualificação das habilidades sociais educativas (práticas positivas), são considerados habilidosos os pais com as seguintes qualidades: que se comunicam de forma assertiva e atenciosa; que fazem perguntas e ouvem seus filhos atentamente em ocasiões oportunas; que expressam sentimentos positivos exaltando a importância do adolescente; que expressam sentimentos negativos de forma assertiva e convenientes a situações ou comportamentos inadequados do adolescente; que expressam opiniões em situações adequadas, respeitando as opiniões alheias e as discordâncias; que fazem carinho; que conseguem estabelecer limites coerentes a situações e à idade do filho sem utilizar ameaças e chantagens e os pais que conseguem dialogar de forma assertiva e agir de forma consistente com seus filhos.

A partir dessa análise, o comportamento dos filhos também pode ser investigado e classificado em habilidoso ou não habilidoso. Habilidoso é caracterizado aquele filho que se dispõe e coopera com a comunicação e resolução de problemas; aquele que expressa suas preferências; que emite críticas construtivas e expressa carinho e empatia. Não habilidoso é o comportamento do filho que enfrenta dificuldades de interação social, como a falta de assertividade, a agressividade, a pouca emissão de carinho e de disponibilidade; que tem atitudes consideradas problemáticas, as quais são classificadas em internalizantes e externalizantes (BOLSONI-SILVA, 2008).

Quanto à identificação de padrões distintos de comportamentos entre o gênero feminino e masculino, pode-se citar o estudo de Camacho e Matos (2006). De acordo com a pesquisa, as adolescentes relataram ter mais supervisão e apoio dos pais, mas também afirmaram ter experimentado álcool e tabaco com maior frequência. Nesse caso, não se pode afirmar que o monitoramento e a supervisão dos pais favoreceram o não consumo de substâncias.

Em direção complementar, Pacheco et al. (2005) concluem que, apesar de estudos indicarem a prevalência de comportamentos antissociais entre os meninos e adolescentes, a estabilidade e a forma como evoluem esses problemas entre o gênero feminino deve ser foco de preocupação. Tais achados sugerem a importância de se estudar diferenças e semelhanças de gênero na população adolescente, bem como associações a práticas parentais.

Os problemas de comportamento do adolescente surgem, por isso, associados ao seu meio social (PATTERSON; REID; DISHION, 2002), em especial, ao contexto familiar. No caso das habilidades sociais educativas parentais aplicadas ao adolescente brasileiro, a literatura é escassa, justificando novas pesquisas. 
Diante dessas considerações, o presente estudo buscou (a) correlacionar as práticas educativas maternas, as habilidades sociais dos adolescentes e os problemas de comportamento, considerando grupos de risco e de não risco para problemas de comportamento; (b) comparar os comportamentos a partir do sexo dos adolescentes.

\section{Método}

Participantes:

Participaram deste estudo 24 mães e seus filhos adolescentes, de ambos os sexos, com idades entre 12 e 16 anos, que estudavam na $7 a$. e $8^{a}$. séries de um colégio estadual de uma das capitais da região sul do país. Dentre as mães, 14 manifestaram práticas de risco para problemas de comportamento em adolescentes e 10 de não risco, segundo o Inventário de Estilo Parental - IEP (GOMIDE, 2006). A amostra de adolescentes foi composta por 10 meninos e 14 meninas no total, dos quais seis meninos e oito meninas fizeram parte do grupo de risco para problemas de comportamento e quatro meninos e seis meninas do grupo de não risco.

A idade dos adolescentes variou dos 12 aos 16 anos de idade. A média de idade dos participantes foi de 14 anos ( $D P=1,06$ ). Em relação às mães, a idade variou entre 30 e 58 anos; logo, a média de idade das participantes foi de 39 anos (DP =6,77).

Quanto aos dados demográficos, os grupos foram equivalentes quanto a: idade dos adolescentes $(p=0,278)$ e das mães $(p=0,32)$; renda familiar mensal $(D P=0,93 / p=0,60)$, esta variou de 1,4 a 7,4 salários mínimos; estado civil materno $(D P=1,18 / p=0,28)$; escolaridade das mães ( $D P=1,56 / p=0,12)$; profissão ou ocupação $(D P=3,09 / p=0,84)$; jornada de trabalho ( $D P=1,1 / p=0,43)$ e vínculo empregatício das mães $(D P=0,48 / p=0,77)$. Esses dados foram avaliados pelo teste CrossTabs.

Procedimentos:

Este estudo foi aprovado pelo Comitê de Ética da Faculdade de Ciências da Universidade Estadual Paulista Júlio de Mesquita Filho (UNESP) do Campus de Bauru (processo no. 1175/46/01/08) e foi autorizado pela Secretaria da Educação do Estado do Paraná. As mães receberam os esclarecimentos sobre a pesquisa e assinaram o Termo de Consentimento Livre Esclarecido. Os adolescentes foram autorizados pelos pais a responder ao IEP em sala de aula coletiva, mas individualmente. Os participantes do estudo foram selecionados a partir das respostas dos adolescentes, ou seja, eles foram escolhidos por relatarem práticas maternas de risco e não risco, que são fatores influentes para que a juventude desenvolva ou não problemas de comportamento.

Critério de inclusão dos participantes: 
No grupo de risco e de não risco para problemas de comportamento, o critério de inclusão dos participantes foi obtido pela aplicação do Inventário de Estilo Parental - IEP - em 276 adolescentes.

Dos 276 inventários tabulados, 144 mães apresentaram índices negativos, o que indica a prevalência das práticas maternas de risco para problemas de comportamento dos filhos, e 24 mães apresentaram índices positivos, o que é indicativo da prevalência das práticas maternas de não risco para problemas de comportamento dos adolescentes ${ }^{2}$.

Foram selecionadas para este estudo, as mães com estilo de risco e as com estilo de não risco para problemas de comportamento dos adolescentes. Com tal critério de inclusão, foram escolhidas 48 mães, das quais 24 são do grupo de risco e 24 do grupo de não risco. A pesquisadora entrou em contato com as mães por telefone para convidá-las a participar da segunda fase da pesquisa. Do total, 14 mães do grupo de risco e 10 mães do de não risco compareceram às entrevistas, compondo a amostra da presente pesquisa.

Instrumentos:

O Inventário de Estilo Parental - IEP (GOMIDE, 2006) é composto por questões sobre as práticas educativas positivas (comportamento moral e monitoria positiva) e sobre as práticas negativas (monitoria negativa, disciplina relaxada, negligência, abuso físico e punição inconsistente). Das 42 questões, há seis para cada prática educativa. As respostas são dispostas em escala likert: sempre (2), às vezes (1) ou nunca $(0)$.

O índice de estilo parental é obtido pela soma das práticas positivas subtraída da soma das negativas. $O$ índice de estilo parental negativo é indicador da prevalência de práticas negativas, as quais se sobrepõem às positivas. O índice de estilo parental menor ou igual a menos dois é classificado como estilo de risco para problemas de comportamento do filho.

O índice de estilo parental materno maior ou igual a onze é considerado estilo de não risco para problemas de comportamento do filho, isto é, indica a forte presença de práticas positivas, as quais são promotoras do comportamento pró-social dos filhos e preventivas de problemas de comportamento dos mesmos. Os índices de estilo medianos, maiores que menos dois e menores que onze, são considerados regulares. O IEP permite o diagnóstico de famílias de risco ou não risco para problemas de comportamento.

O Roteiro de Entrevista de Habilidades Sociais Educativas Parentais RE-HSE-P (BOLSONI-SILVA, 2009) possibilita a análise das interações entre pais e filhos pela investigação de três categorias de habilidades sociais educativas parentais: comunicação, expressão de sentimentos e enfrentamento e estabelecimento de limites. A partir dessas três grandes categorias, avaliam-se as habilidades sociais educativas 
parentais (práticas positivas), as práticas negativas parentais, as habilidades sociais e os problemas de comportamento dos filhos.

O Child Behavior Checklist - CBCL (ACHENBACH, 1991) é um inventário em que as mães ou os pais relatam os comportamentos emitidos por seus filhos. O CBCL é utilizado para o diagnóstico do comportamento de crianças e adolescentes de 6 a 18 anos de idade. 0 instrumento permite a avaliação de dois âmbitos: o da competência social e o dos problemas de comportamento do jovem.

O repertório comportamental infanto-juvenil é classificado em clínico, borderline e não clínico. A amostra clínica é composta por participantes que apresentam distúrbios de comportamento já instaurados; a borderline por adolescentes com problemas de comportamento, considerados de risco para o desenvolvimento de distúrbios; e o grupo não clínico apresenta repertório comportamental considerado competente no meio social, podendo haver alguns problemas de comportamento, mas a frequência e composição de comportamentos não são considerados de risco para distúrbios delinquentes e agressivos (externalizantes) ou depressivos e ansiosos (internalizantes). Nesta pesquisa, utilizou-se apenas a escala de problemas de comportamento.

Tratamento dos dados:

Para avaliação estatística dos dados, utilizou-se o programa estatístico SPSS 10 for Windows. A busca de correlações entre as variáveis do IEP, para cada grupo (de risco e não risco), foi realizada através da aplicação do teste Spearman. Esse procedimento viabilizou a identificação da existência ou não de diferenças internas dentro de cada grupo.

A comparação entre os grupos quanto às habilidades sociais educativas maternas (práticas positivas), às práticas negativas maternas, às habilidades sociais e aos problemas de comportamento do adolescente foi realizada através do teste de Mann-Whitney. A busca de correlações entre as variáveis do RE-HSE-P para cada grupo (de risco e não risco) foi realizada através da aplicação do teste Spearman.

Para a análise dos dados referentes ao Child Behavior Checklist $\mathrm{CBCL}$ (ACHENBACH, 1991) aplicou-se o teste de Mann-Whitney para comparar os resultados do grupo de risco e o de não risco em relação aos problemas de comportamento dos adolescentes.

\section{Resultados}

Com a intenção de verificar a relação entre os comportamentos maternos e filiais avaliados pelos diversos instrumentos, a seção de resultados apresenta as correlações obtidas entre o IEP, O RE-HSE-P e o CBCL. Em seguida, apresentam-se as comparações entre os grupos de risco e não risco quanto às habilidades sociais educativas 
maternas e aos comportamentos dos adolescentes. Por último, pretende-se comparar os resultados das práticas aplicadas aos adolescentes do sexo feminino e masculino, bem como investigar as habilidades sociais e os problemas de comportamento na população adolescente masculina e feminina deste estudo.

Os resultados obtidos pelas correlações entre as habilidades sociais educativas maternas, avaliadas pelo RE-HSE-P; as práticas educativas maternas, investigadas pelo IEP; as habilidades sociais, verificadas pelo RE-HSE-P; os problemas de comportamento dos filhos adolescentes, explorados pelo $\mathrm{CBCL}$ são apresentados na Tabela 1:

\begin{tabular}{|c|c|c|c|c|c|c|}
\hline $\begin{array}{l}\text { Correlação não } \\
\text { paramétrica- } \\
\text { Spearman }\end{array}$ & $\begin{array}{l}\text { Habilidades } \\
\text { sociais } \\
\text { educativas } \\
\text { maternas - } \\
\text { práticas } \\
\text { positivas } \\
\text { (RE-HSE-P) }\end{array}$ & $\begin{array}{l}\text { Práticas } \\
\text { negativas } \\
\text { maternas } \\
\text { (RE-HSE-P) }\end{array}$ & $\begin{array}{l}\text { Habilidades } \\
\text { sociais dos } \\
\text { adolescentes } \\
\text { (RE-HSE-P) }\end{array}$ & $\begin{array}{l}\text { Problemas } \\
\text { internalizantes } \\
\text { dos } \\
\text { adolescentes } \\
(\mathrm{CBCL})\end{array}$ & $\begin{array}{l}\text { Problemas } \\
\text { externalizantes } \\
\text { dos } \\
\text { adolescentes } \\
(\mathrm{CBCL})\end{array}$ & $\begin{array}{l}\text { Total de } \\
\text { problemas de } \\
\text { comportamen } \\
\text { to dos } \\
\text { adolescentes } \\
(\mathrm{CBCL})\end{array}$ \\
\hline $\begin{array}{l}\text { Habilidades } \\
\text { sociais } \\
\text { educativas } \\
\text { maternas - } \\
\text { práticas } \\
\text { positivas (RE- } \\
\text { HSE-P) }\end{array}$ & - & - & $\begin{array}{l}0,921^{* *} \\
, 000\end{array}$ & $\begin{array}{l}-, 493^{*} \\
, 014\end{array}$ & $\begin{array}{l}-, 515^{*} \\
, 010\end{array}$ & $\begin{array}{l}-, 601^{* * *} \\
, 002\end{array}$ \\
\hline $\begin{array}{l}\text { Práticas } \\
\text { negativas } \\
\text { maternas } \\
\text { (RE-HSE-P) }\end{array}$ & $=$ & - & $\begin{array}{l}-, 519^{* \ldots} \\
0,009\end{array}$ & $\begin{array}{l}.420^{*} \\
.041\end{array}$ & $\begin{array}{l}839^{* * 3} \\
, 000\end{array}$ & $\begin{array}{l}822^{* * *} \\
, 000\end{array}$ \\
\hline $\begin{array}{l}\text { Habilidades } \\
\text { sociais dos } \\
\text { adolescentes } \\
\text { (RE-HSE-P) }\end{array}$ & $\begin{array}{l}0,921^{* *} \\
, 000\end{array}$ & $\begin{array}{l}-, 519^{* *} \\
0,009\end{array}$ & - & $\begin{array}{l}-, 413^{*} \\
045\end{array}$ & $\begin{array}{l}-, 572^{* *} \\
, 003\end{array}$ & $\begin{array}{l}-, 614^{* * *} \\
, 001\end{array}$ \\
\hline $\begin{array}{l}\text { Indice de estilo } \\
\text { parental (IEP) }\end{array}$ & $\begin{array}{l}787^{* * *} \\
000\end{array}$ & $\begin{array}{l}-, 668^{* * *} \\
, 000\end{array}$ & $\begin{array}{l}733^{* * *} \\
000\end{array}$ & $\begin{array}{l}-, 490^{*} \\
, 015\end{array}$ & $\begin{array}{l}-, 651^{* * *} \\
, 001\end{array}$ & $\begin{array}{l}-, 712^{* * *} \\
, 000\end{array}$ \\
\hline $\begin{array}{l}\text { Monitoria } \\
\text { positiva } \\
\text { materna (IEP) }\end{array}$ & $\begin{array}{l}690^{* * \cdots} \\
000\end{array}$ & $\begin{array}{l}-, 604^{* \ldots} \\
, 002\end{array}$ & $\begin{array}{l}614^{* * *} \\
001\end{array}$ & $\begin{array}{l}-, 531^{\cdots *} \\
, 008\end{array}$ & $\begin{array}{l}-, 554^{* *} \\
, 005\end{array}$ & $\begin{array}{l}-, 603^{\text {.6. }} \\
, 002\end{array}$ \\
\hline $\begin{array}{l}\text { Comportament } \\
\text { o moral } \\
\text { materno (IEP) }\end{array}$ & $\begin{array}{l}678^{\cdots \cdots} \\
000\end{array}$ & $\begin{array}{l}-, 421^{*} \\
, 041\end{array}$ & $\begin{array}{l}648^{* * *} \\
000\end{array}$ & $\begin{array}{l}-, 537^{3 *} \\
, 007\end{array}$ & $\begin{array}{l}-, 566^{* 37} \\
, 004\end{array}$ & $\begin{array}{l}-, 558^{\ldots \ldots} \\
, 005\end{array}$ \\
\hline $\begin{array}{l}\text { Puniçầo } \\
\text { inconsistente } \\
\text { materna (IEP) }\end{array}$ & $\begin{array}{l}-, 694^{* 3} \\
, 000\end{array}$ & $\begin{array}{l}, 735^{7 \%} \\
, 000\end{array}$ & $\begin{array}{l}-, 729^{* *} \\
, 000\end{array}$ & $\begin{array}{l}351 \\
, 093\end{array}$ & $\begin{array}{l}, 712 \\
, 000\end{array}$ & $\begin{array}{l}, 694^{* * 3} \\
, 000\end{array}$ \\
\hline $\begin{array}{l}\text { Disciplina } \\
\text { relaxada } \\
\text { materna (IEP) }\end{array}$ & $\begin{array}{l}-, 511^{* 3} \\
, 011\end{array}$ & $\begin{array}{l}, 418^{*} \\
, 042\end{array}$ & $\begin{array}{l}-, 605^{\ldots *} \\
, 002\end{array}$ & $\begin{array}{l}.079 \\
.713\end{array}$ & $\begin{array}{l}577^{* 3 *} \\
, 003\end{array}$ & $\begin{array}{l}509^{*} \\
, 011\end{array}$ \\
\hline $\begin{array}{l}\text { Monitoria } \\
\text { negativa } \\
\text { materna (IEP) }\end{array}$ & $\begin{array}{l}-, 671^{* 3} \\
, 000\end{array}$ & $\begin{array}{l}672 \\
, 000\end{array}$ & $\begin{array}{l}-, 628^{\cdots *} \\
, 001\end{array}$ & $\begin{array}{l}394 \\
, 057\end{array}$ & $\begin{array}{l}526^{3 * 3} \\
, 008\end{array}$ & $\begin{array}{l}537^{* * *} \\
, 007\end{array}$ \\
\hline $\begin{array}{l}\text { Negligência } \\
\text { materna (IEP) }\end{array}$ & $\begin{array}{l}-, 661^{* *} \\
, 000\end{array}$ & $\begin{array}{l}593^{\cdots *} \\
, 002\end{array}$ & $\begin{array}{l}-, 607^{\cdots *} \\
, 002\end{array}$ & $\begin{array}{l}, 358 \\
, 086\end{array}$ & $\begin{array}{l}505^{*-} \\
, 012\end{array}$ & $\begin{array}{l}, 538^{* * *} \\
, 007\end{array}$ \\
\hline $\begin{array}{l}\text { Abuso fisico } \\
\text { materno (IEP) }\end{array}$ & $\begin{array}{l}-, 639^{3 * *} \\
, 001\end{array}$ & $\begin{array}{l}, 607^{* *} \\
, 002 \\
\end{array}$ & $\begin{array}{l}-, 492^{* * *} \\
, 015 \\
\end{array}$ & $\begin{array}{l}314 \\
, 136 \\
\end{array}$ & $\begin{array}{l}, 395 \\
, 056 \\
\end{array}$ & $\begin{array}{l}489^{*} \\
015\end{array}$ \\
\hline
\end{tabular}


Os resultados apresentados na Tabela 1 mostram que houve correlação positiva entre o total de habilidades sociais educativas maternas (práticas positivas), verificado pelo RE-HSE-P, e o índice de estilo parental do IEP. Esses resultados indicam que quanto maior a frequência de habilidades sociais educativas maternas do RE-HSE-P, maiores são os índices de estilo parental do IEP, os quais indicam a maior frequência de práticas positivas no repertório materno. A correlação negativa foi detectada entre o total de práticas negativas do RE-HSE-P e o índice de estilo parental (IEP). O resultado indica que há associação entre a maior frequência de práticas negativas do RE-HSE-P e a menor a frequência de índices de estilo parental positivos do IEP.

O total de habilidades sociais educativas maternas (práticas positivas) do RE-HSE-P obteve correlações positivas com todas as práticas educativas positivas do IEP (monitoria positiva e comportamento moral). Já as habilidades sociais educativas maternas, do RE-HSE-P, apresentaram correlações negativas com as práticas negativas de punição inconsistente, disciplina relaxada, negligência, monitoria negativa e abuso físico materno, avaliadas pelo IEP.

O total de práticas negativas do RE-HSE-P obteve correlação negativa com todas as práticas educativas positivas do IEP (monitoria positiva e comportamento moral). As práticas negativas, do RE-HSE-P, por sua vez, apresentaram correlações positivas com todas as práticas negativas do IEP: punição inconsistente, disciplina relaxada, negligência, monitoria negativa e abuso físico materno.

Constatou-se correlação positiva entre as habilidades sociais educativas maternas (práticas positivas maternas) e as habilidades sociais dos adolescentes. Houve também correlação negativa entre as práticas negativas maternas, avaliadas pelo RE-HSE-P, e as habilidades sociais dos filhos adolescentes, investigadas pelo mesmo instrumento.

Também houve correlação negativa entre as habilidades sociais educativas maternas (RE-HSE-P) e os problemas de comportamento internalizantes, externalizantes e 0 total de problemas de comportamento dos adolescentes, investigados pelo CBCL. O contrário ocorreu entre as práticas negativas, do RE-HSE-P, e os problemas de comportamento dos filhos, situação em que, quanto maior a frequência de práticas negativas maternas, maior foi a frequência de problemas internalizantes, externalizantes e o total de problemas de comportamento dos adolescentes.

As variáveis maternas, avaliadas pelo IEP, indicaram que todas as práticas maternas estão correlacionadas às habilidades sociais e ao total de problemas de comportamento dos filhos adolescentes. As práticas positivas estiveram correlacionadas positivamente às habilidades sociais dos adolescentes, avaliadas pelo RE-HSE-P, e negativamente ao total de problemas de comportamento, aos 
problemas internalizantes e aos externalizantes dos adolescentes, investigados pelo CBCL.

Quanto às práticas maternas negativas, avaliadas pelo IEP, correlações positivas foram obtidas entre as práticas negativas: disciplina relaxada, monitoria negativa, negligência e os problemas de comportamento externalizantes dos adolescentes. Não foram encontradas correlações entre as práticas maternas negativas do IEP e os problemas de comportamento internalizantes.

O índice de estilo parental do IEP obteve correlações negativas com o total de problemas de comportamento, com os problemas internalizantes e externalizantes dos filhos.

A comparação entre os grupos de risco e os de não risco em relação às habilidades sociais educativas maternas, às habilidades sociais e aos problemas de comportamento dos adolescentes aparece na Tabela 2:

Tabela 2 - Comparação entre os grupos de risco e os de não risco quanto às habilidades sociais educativas maternas, às habilidades sociais e aos problemas de comportamento dos adolescentes.

\begin{tabular}{|c|c|c|c|}
\hline Variáveis do RE-HSE-P e do CBCL & $\begin{array}{l}\text { Média do } \\
\text { grupo de } \\
\text { risco }\end{array}$ & $\begin{array}{l}\text { Média do } \\
\text { grupo de } \\
\text { não risco }\end{array}$ & $\begin{array}{l}\text { Comparação entre os } \\
\text { grupos - Mann Whitney } \\
\text { test }\end{array}$ \\
\hline $\begin{array}{l}\text { Total de habilidades sociais educativas matemas } \\
\text { - práticas positivas matemas (RE-HSE-P) }\end{array}$ & 8,14 & 18,6 & $p=0,000$ \\
\hline $\begin{array}{l}\text { Total de práticas negativas matemas } \\
\text { (RE-HSE-P) }\end{array}$ & 16,79 & 6,5 & $p=0,000$ \\
\hline Habilidades sociais dos adolescentes (RE-HSE-P) & 8,21 & 18,5 & $\mathrm{P}=0,000$ \\
\hline $\begin{array}{l}\text { Problemas intemalizantes dos adolescentes } \\
(\mathrm{CBCL})\end{array}$ & 14,96 & 9,05 & $\mathrm{p}=0,043$ \\
\hline $\begin{array}{l}\text { Problemas extemalizantes dos adoles centes } \\
\text { ( } \mathrm{CBCL} \text { ) }\end{array}$ & 16,75 & 6,55 & $p=0,000$ \\
\hline $\begin{array}{l}\text { Total de problemas de comportamento dos } \\
\text { adolescentes } \\
\text { ( } \mathrm{CBCL} \text { ) }\end{array}$ & 16,79 & 6,5 & $p=0,000$ \\
\hline
\end{tabular}

Observando a Tabela 2, percebe-se que houve diferenças estatísticas significativas entre os grupos para todos os quesitos investigados. Nota-se que as habilidades sociais educativas maternas (práticas positivas) foram mais utilizadas pelas mães do grupo de não risco do que pelas mães do grupo de risco, com diferença estatística significativa $(p=0,00)$. Já as práticas negativas foram mais frequentes entre as mães do grupo de risco do que entre as do grupo de não risco, com diferença estatística significativa $(p=0,00)$.

Os adolescentes do grupo de risco apresentaram menos habilidades sociais e mais problemas de comportamento internalizantes $(p=0,04)$, externalizantes $(p=0,00)$ e maior frequência no total de problemas de comportamento $(p=0,00)$ do que os adolescentes do grupo de não risco, com diferenças estatísticas significativas para todos os quesitos avaliados. A comparação entre os adolescentes do sexo feminino e masculino no que se refere às práticas maternas, às 
habilidades sociais educativas maternas, às habilidades sociais e aos problemas de comportamento dos adolescentes aparece na Tabela 3:

Tabela 3: Comparação entre os adolescentes do sexo feminino e masculino quanto às práticas educativas (IEP), às habilidades sociais educativas maternas (RE-HSEP) e as habilidades sociais (RE-HSE-P) e os problemas de comportamento dos adolescentes (CBCL).

\begin{tabular}{|c|c|c|c|c|c|}
\hline \multicolumn{2}{|c|}{ Variáveis: IEP, RE-HSE-P e CBCL } & \multirow{2}{*}{$\begin{array}{l}\text { Gênero } \\
\text { Masculino }\end{array}$} & \multirow{2}{*}{$\begin{array}{l}\text { Número de } \\
\text { participantes } \\
10\end{array}$} & \multirow{2}{*}{$\begin{array}{l}\text { Médias } \\
12,05\end{array}$} & \multirow{2}{*}{$\begin{array}{l}\text { Mann } \\
\text { Whitney } \\
0,790\end{array}$} \\
\hline \multirow[t]{16}{*}{ IEP } & \multirow[t]{2}{*}{ Monitoria positiva } & & & & \\
\hline & & Feminino & 14 & 12,82 & \\
\hline & \multirow[t]{2}{*}{ Comportamento moral } & Masculino & 10 & 12,5 & \multirow[t]{2}{*}{1,000} \\
\hline & & Feminino & 14 & 12,5 & \\
\hline & \multirow[t]{2}{*}{ Punição inconsistente } & Masculino & 10 & 11,1 & \multirow[t]{2}{*}{0,409} \\
\hline & & Feminino & 14 & 13,5 & \\
\hline & \multirow[t]{2}{*}{ Negligência } & Masculino & 10 & 11,55 & \multirow[t]{2}{*}{0,570} \\
\hline & & Feminino & 14 & 13,18 & \\
\hline & \multirow[t]{2}{*}{ Disciplina relaxada } & Masculino & 10 & 12 & \multirow[t]{2}{*}{0,768} \\
\hline & & Feminino & 14 & 12,8 & \\
\hline & \multirow[t]{2}{*}{ Monitoria negativa } & Masculino & 10 & 12,95 & \multirow[t]{2}{*}{0,790} \\
\hline & & Feminino & 14 & 12,18 & \\
\hline & \multirow[t]{2}{*}{ Abuso físico } & Masculino & 10 & 11,9 & \multirow[t]{2}{*}{0,720} \\
\hline & & Feminino & 14 & 12,93 & \\
\hline & \multirow{2}{*}{$\begin{array}{l}\text { Indice de estilo parental } \\
\text { (IEP) }\end{array}$} & Masculino & 10 & 12,75 & \multirow[t]{2}{*}{0,883} \\
\hline & & Feminino & 14 & 12,32 & \\
\hline \multirow{6}{*}{$\begin{array}{l}\text { RE- } \\
\text { HSE-P }\end{array}$} & \multirow{2}{*}{$\begin{array}{l}\text { Total de práticas positivas } \\
\text { maternas }\end{array}$} & Masculino & 10 & 13,2 & \multirow[t]{2}{*}{0,681} \\
\hline & & Feminino & 14 & 12 & \\
\hline & \multirow{4}{*}{$\begin{array}{l}\text { Total de praticas negativas } \\
\text { maternas } \\
\text { Habilidades sociais dos } \\
\text { adolescentes }\end{array}$} & Masculino & 10 & 13,8 & \multirow[t]{2}{*}{0,445} \\
\hline & & Feminino & 14 & 11,57 & \\
\hline & & Masculino & 10 & 13,45 & 0,577 \\
\hline & & Feminino & 14 & 11,82 & \\
\hline \multirow[t]{6}{*}{$\mathrm{CBCL}$} & \multirow{2}{*}{$\begin{array}{l}\text { Problemas intermalizantes } \\
\text { dos adolescentes }\end{array}$} & Masculino & $10^{\circ}$ & 14 & \multirow[t]{2}{*}{0,379} \\
\hline & & Feminino & 14 & 11,43 & \\
\hline & \multirow{2}{*}{$\begin{array}{l}\text { Problemas extemalizantes } \\
\text { dos adolescentes }\end{array}$} & Masculino & 10 & 14,7 & \multirow[t]{2}{*}{0,196} \\
\hline & & Feminino & 14 & 10,93 & \\
\hline & \multirow{2}{*}{$\begin{array}{l}\text { Total de problemas de } \\
\text { comportamento dos } \\
\text { adolescentes }\end{array}$} & Masculino & 10 & 14,85 & 0,168 \\
\hline & & Feminino & 14 & 10,82 & \\
\hline
\end{tabular}

Como mostra a Tabela 3, não há diferenças entre os grupos de meninos e meninas participantes em relação a todas as variáveis investigadas. Esse resultado indica que nesta pesquisa não houve diferenças significativas entre as práticas e as habilidades sociais educativas maternas aplicadas aos meninos e às meninas. Também não houve diferenças significativas entre as habilidades sociais e os problemas de comportamento apresentados pelos adolescentes do sexo feminino e masculino.

\section{Discussão}

Neste estudo, as habilidades sociais educativas maternas, investigadas pelo RE-HSE-P, estiveram positivamente correlacionadas com as práticas educativas e com os índices de estilo materno positivos, avaliados pelo IEP. Sendo assim, permite-se afirmar que os instrumentos foram convergentes em seus achados, o que aumenta a 
consistência dos dados encontrados. Nesse sentido, o método correlacional mostra-se um eficiente recurso para tal fim (DANCEY; REIDY, 2006), conforme outros estudos (GOMIDE et al., 2006; SALVO; SILVARES; TONI, 2005) apontam.

As habilidades sociais educativas maternas foram positivamente correlacionadas com as habilidades sociais dos adolescentes, o que possivelmente indica que aspectos da interação entre as mães e os filhos estimulam as habilidades sociais dos mesmos. Resultados semelhantes foram encontrados por Gomide et al. (2006) ao detectarem que pais, mães e filhos adolescentes de famílias de não risco apresentavam mais habilidades sociais de expressividade do que os de famílias de risco.

Segundo Stattin e Kerr (2000), quando os pais e as mães conseguem estabelecer uma comunicação habilidosa, concomitante a regras consistentes, os filhos se apresentam mais abertos à comunicação e mais habilidosos nas situações cotidianas. Teixeira, Oliveira e Wottrich (2006), ao estudarem a percepção dos adolescentes sobre seus pais, perceberam que os pais que supervisionavam e ao mesmo tempo apoiavam emocionalmente seus filhos eram percebidos por estes como estimuladores da comunicação e da autonomia. Já aqueles que se sentiam coagidos pelos pais manifestavam medo de expressar os sentimentos e de fazer escolhas por conta própria. Portanto, os estudos prévios apontam para a relação entre as habilidades sociais parentais e as habilidades sociais dos filhos, relação também encontrada neste estudo.

As variáveis maternas consideradas habilidosas foram negativamente correlacionadas com problemas de comportamento internalizantes, externalizantes e com o total de problemas dos adolescentes avaliados pelo $C B C L$, o que confirma os estudos da área (AVANCI et al., 2007; SALVO; SILVARES; TONI, 2005; FLETCHER; STEINBERG; WILLIAMS-WHEELER, 2004). O resultado está em consonância com o estudo que tem como base a revisão da literatura sobre o uso de drogas na adolescência (SCHENKER; MINAYO, 2005). Nessa pesquisa, foram apontados alguns fatores protetores do adolescente. Dos fatores preventivos, aparece a família que apoia a aquisição da autonomia do filho, monitora e estabelece normas claras para comportamento dos adolescentes (SCHENKER; MINAYO, 2005).

Tanto Pacheco, Silveira e Schneider (2008) quanto Reichert e Wagner (2007) apoiam a ideia de que, na adolescência, os pais devem estimular a autonomia dos filhos através de comportamentos responsivos. Entre os comportamentos estão a aceitação e a divisão na tomada de decisão, os aspectos coerentes com a habilidade de comunicação, a expressividade e a monitoria positiva. Tais comportamentos parentais podem evitar a ocorrência de problemas como a depressão e a ansiedade (GOMIDE, 2006; BOLSONI-SILVA, 2009). 
Em congruência à associação das práticas positivas e do comportamento socialmente habilidoso do filho, uma pesquisa sobre a saúde do adolescente (DICLEMENTE et al., 2001) percebeu que há índices menores de doenças sexualmente transmissíveis em jovens cujos pais monitoram positivamente seus filhos através da comunicação. Já os achados sobre as práticas negativas maternas, no RE-HSE-P e no IEP, foram associados aos problemas de comportamento dos adolescentes. Esses dados estão de acordo com os estudos prévios, como o de Avanci et al. (2007) que encontrou associação entre as práticas parentais abusivas e os transtornos psiquiátricos depressivos e ansiosos dos adolescentes.

As práticas abusivas foram descritas pelos autores (AVANCI et al., 2007) como cobranças parentais excessivas que são responsáveis pela redução e desvalorização das capacidades, dos pensamentos, das vontades e das emoções dos filhos. Essa relação também foi detectada pelo presente estudo como uma associação entre a monitoria negativa e os problemas de comportamento inter e externalizantes dos adolescentes.

Schenker e Minayo (2005) descreveram algumas práticas parentais de risco para o problema de envolvimento do adolescente com as drogas. Para as autoras, a falta de vínculo e de interação dos pais com os filhos, caracterizada como negligência, está associada a problemas de comportamento do adolescente. Elas também citaram a falta de consistência das práticas parentais, a permissividade, como a falta de aplicação e de cumprimento das regras, associada ao problema de comportamento do adolescente. Tais fatos também foram constatados pelo presente estudo, tendo em vista que os altos índices de negligência, disciplina relaxada, punição inconsistente (IEP) e de falta de habilidades na aplicação de limites (RE-HSE-P) estão associados aos problemas de comportamento inter e externalizantes dos adolescentes.

Outros estudos fazem associações entre as práticas parentais e os problemas de comportamento do adolescente relacionados à sexualidade e ao uso de substâncias psicoativas. Os adolescentes que não recebem orientações parentais de forma assertiva, ou que são negligenciados pelos pais, estão mais propensos a envolver-se em situações de risco, como fazer sexo desprotegido e ter vários parceiros sexuais (DICLEMENTE et al., 2001; ROMER, 1994). Sob a influência de tais práticas negativas, os adolescentes também estão mais propensos ao uso de álcool e tabaco (BORAWSKI et al., 2003).

Os resultados deste estudo encontraram correlações negativas entre o Índice de Estilo Parental (IEP) e as variáveis do $C B C L$, de modo que quanto mais alto o índice de estilo materno menor a frequência de problemas de comportamento internalizantes e externalizantes dos filhos adolescentes. Os achados são condizentes à pesquisa de Salvo, Silvares e Toni (2005). Nesse estudo, eles também utilizaram esses 
mesmos instrumentos e fizeram essa correlação. Os resultados são congruentes também com o estudo de Gomide et al. (2006). Nesta pesquisa, cujos pais e as mães apresentaram altos índices de estilo parental, os filhos não apresentaram sintomas de depressão.

Este estudo também buscou a comparação entre os grupos de risco e os de não risco quanto às médias das habilidades sociais educativas maternas (práticas positivas), às práticas negativas maternas e à frequência de habilidades sociais e problemas de comportamento dos adolescentes. O resultado apontou para maior frequência de habilidades sociais educativas maternas no grupo de mães de não risco do que no grupo materno de risco para problemas de comportamento do adolescente, sugerindo o mesmo padrão encontrado em pré-escolares (BOLSONI-SILVA; MARTURANO, 2008).

Os adolescentes do grupo de risco apresentaram maior frequência de problemas de comportamento, sejam internalizantes ou externalizantes, que os do grupo de não risco, confirmando os achados de Gomide et al. (2006). O estudo mostra que os pais e as mães com altos níveis de habilidades sociais, avaliados pelo IHS (DEL PRETTE; DEL PRETTE, 2000), aplicam mais práticas educativas positivas, e os seus filhos apresentam menos problemas como stress, depressão e mais habilidades sociais.

Os resultados deste estudo apontam para a não diferença entre as práticas maternas aplicadas aos meninos e as meninas, bem como a ausência de divergências entre problemas de comportamento em adolescentes do sexo feminino e masculino, em consonância com preocupações sinalizadas por Camacho e Matos (2006) e por Pacheco et al. (2005).

No caso de população pré-escolar, escolar e adolescente os resultados quanto ao gênero, considerando a sequência desenvolvimentista (PATTERSON; REID; DISHION, 2002) não são conclusivos. Nesse sentido, os resultados obtidos no presente estudo estão de acordo com Marturano, Toller e Elias (2005) que afirmam que não há maior frequência de problemas de comportamento na população masculina em relação à feminina.

Alguns achados desta pesquisa, no entanto, divergem do estudo realizado por Maturano, Toller e Elias (2005), visto que os autores apontaram para as diferenças qualitativas entre os problemas de comportamento masculinos e femininos. As meninas desse caso apresentaram maior frequência de problemas de comportamento internalizantes, como ansiedade, depressão e queixas somáticas. 0 contrário ocorreu no presente estudo, pois não foram detectadas diferenças quanto ao tipo de problemas de comportamento em adolescentes do sexo feminino e masculino. Em outras palavras, os meninos e as meninas apresentaram problemas de comportamento internalizantes e externalizantes em frequências semelhantes. 


\section{Considerações finais}

Os achados deste estudo apontam que as práticas positivas maternas, investigadas a partir de dois instrumentos distintos, estão relacionadas à menor incidência de problemas de comportamento e à maior frequência de habilidades sociais em adolescentes. Não foram encontradas diferenças entre as práticas maternas e os comportamentos dos filhos no que se refere ao gênero.

Detectou-se que as mães do grupo de não risco utilizaram mais habilidades sociais educativas, o que significa que se comunicaram, expressaram sentimentos e aplicaram limites de forma mais habilidosa. Tal comportamento materno habilidoso pode ser considerado como um dos fatores que possibilitou aos filhos adolescentes a maior frequência de habilidades sociais e a menor de problemas de comportamento. O contrário se deu no grupo de risco, em que as mães agiram de forma menos habilidosa e aplicaram mais práticas negativas. Agindo assim, seus filhos apresentaram menores índices de habilidades sociais e maiores de problemas de comportamento.

Tais constatações vêm ressaltar a importância dos grupos de apoio e de orientação para os pais como forma de prevenção de problemas de comportamento dos filhos adolescentes (BOLSONI-SILVA, 2007). Esse suporte pode possibilitar aos pais a detecção de suas práticas e o aprimoramento delas, conforme proposto por estudos da área (PINHEIRO et al., 2006; BIASOLI-ALVES, 2005; TEIXEIRA, 1996), destacando que as práticas parentais devem estar em consonância com a fase de desenvolvimento dos filhos.

Outra proposta é a intervenção voltada para a estimulação das habilidades sociais dos filhos, concomitante à orientação dos pais (BARALDI; SILVARES, 2003). A intervenção é um recurso que possibilita a estimulação das habilidades sociais dos pais e a dos filhos, bem como previne problemas de comportamento em adolescentes. Tendo em vista que a ausência ou pouca frequência das habilidades sociais educativas parentais pode influenciar 0 surgimento de problemas de comportamento nos filhos (BOLSONISILVA; MARTURANO, 2007).

Esta pesquisa possibilitou ampliar o conhecimento acerca da interação entre as práticas educativas parentais e os comportamentos dos filhos adolescentes. Devem, entretanto, ser consideradas algumas limitações do estudo como o número reduzido de participantes e a não aleatoriedade na composição dos dois grupos. Outro aspecto foi o uso de instrumentos de relato, de modo que é ideal o uso concomitante do relato e das medidas diretas no comportamento, o que pode ser considerado em estudos futuros. 


\section{Referências Bibliográficas}

ACHENBACH, T. M.; EDELBROCK, C. S. The child behavior profile: II. Boys aged $12-16$ and girls aged $6-11$ and 12-16. Journal of consulting and clinical psychology, v. 47, n. 2, p. 223-233, 1979. $A C H E N B A C H, T$. M. Manual for the youth self-report and 1991 profile. Burlington: University of Vermont, Departament of psychiatry, 1991.

AVANCI, J.; ASSIS, S. G.; OLIVEIRA, R. V. C; FERREIRA, R. M.; PESCE, R. P. Fatores associados aos problemas de saúde mental em adolescentes. Psicologia: teoria e pesquisa, Brasília, v. 23, n. 3, p. 287-294, 2007.

BARALDI, D. M.; SILVARES, E. F. M. Treino de habilidades sociais em grupo com crianças agressivas, associado à orientação dos pais: análise empírica de uma proposta de atendimento. In: DEL PRETTE, A.; DEL PRETTE, Z. A. P. (Orgs.). Habilidades sociais, desenvolvimento e aprendizagem: questões conceituais, avaliação e intervenção. Campinas: Alínea, 2003, p. 235-258.

BIASOLI-ALVES, Z. M. M. Orientação de pais: partilhar conhecimentos sobre desenvolvimento e práticas de educação como estratégia de intervenção. Texto $\boldsymbol{\&}$ contexto enfermagem, Florianópolis, v. 14, p. 64-70, 2005.

BOLSONI-SILVA, A. T.; MARTURANO, E. M. A qualidade da interação positiva e da consistência parental na sua relação com problemas de comportamentos pré-escolares, habilidades sociais educativas parentais e problemas de comportamento. Revista interamericana de psicologia/ Interamerican journal of psychology, Porto Alegre, v. 41, n. 3, p. 2-10, 2007.

BOLSONI-SILVA, A. T. Roteiro de entrevista de habilidades sociais educativas parentais adaptação de Bolsoni-Silva 2007, não publicado, 2007.

- Roteiro de entrevista de habilidades sociais educativas parentais (RE-HSE-P): categorias e testagens preliminares. In: Weber, L. N. D. (Org.). Família e desenvolvimento humano. Curitiba: Juruá, 2008, p. 145- 158.

- Estudos de validação e confiabilidade do roteiro de entrevista de habilidades sociais educativas parentais (REHSE-P) Tese de pós-doutorado apresentada à Faculdade de Filosofia, Ciências e Letras de Ribeirão Preto da USP. Ribeirão Preto, 108p., 2009.

BORAWSKI, E.; IEVENS-LANDIS, C. E.; LOVERGREEN, L. D.; TRAPI, E. S. Parental monitoring, negotiated unsupervised time, and parental trust: the role of perceived parenting practices in adolescent health risk behaviors. Journal of adolescent health, Deerfield, v. 33, p. 60-70, 2003.

CABALLO, V. Manual de técnicas de terapia e modificação do comportamento. São Paulo: Santos, 2002. 
CAMACHO, I; MATOS, M. G. Práticas parentais, escola e consumo de drogas. Psicologia, saúde \& doenças, Lisboa, v. 7, n. 2, p. 317327, 2006.

DANCEY, C. P.; REIDY, J. Estatística sem matemática para psicologia: usando SPSS para Windows, 3.ed. Porto Alegre: Artmed, 2006.

DEL PRETTE, A.; DEL PRETTE, Z. A. P. Psicologia das habilidades sociais: Terapia e educação. Petrópolis: Vozes, 1999.

I nventário de habilidades sociais Del Prette. São Paulo:

Casa do psicólogo, 2000.

DICLEMENTE, R. J.; WINGOOD, G. M.; CROSBY, R.; SIONEAN, C.; COBB, B. K.; HARRINGTON, K.; DAVIES, S. ; HOOK, E. W. III; OH, M. $\mathrm{K}$. Parental monitoring: association with adolescents' risk behaviors. Pediatrics, Elk Grove Village, v. 10, n. 7, p. 1363-1368, 2001.

FLETCHER, A. C.; STEINBERG, L; WILLIAMS-WHEELER, M. Parental influences on adolescent problem behavior: revisiting Stattin and Kerr. Child development, Raleigh, v. 75, n. 3, p. 781-796, 2004.

GOMIDE, P. I. C. Estilos Parentais e comportamento anti-social. In: DEL PRETTE, A.; DEL PRETTE, Z. (Orgs.). Habilidades Sociais, desenvolvimento e aprendizagem: questões conceituais, avaliação e intervenção. Campinas: Alínea, 2003, p. 21-60.

Pais presentes, pais ausentes. Petrópolis: Vozes, 2004.

Manual do inventário de estilos parentais: modelo teórico, manual de aplicação, apuração e interpretação. Petrópolis: Vozes, 2006.

. SALVO, C. G.; PINHEIRO, D. D.; SABBAG, G. M. Correlação entre práticas educativas, depressão, estresse e habilidades sociais. Psico-USF, Iataiba, v. 10, n. 2, p. 169-178, 2006.

MARTURANO; E. M.; TOLLER, G. P.; ELIAS, L. C. S. Gênero, adversidade e problemas socioemocionais associados à queixa escolar. Estudos de psicologia, Campinas, v. 22, n. 4, p. 371-380, 2005.

MEYER, S. B.; OSHIRO, C.; DONADONE, J. C.; MAYER, R. C. F.; STARLING, R. Subsídios da obra "Comportamento Verbal" de B. F. Skinner para a terapia analítico-comportamental. Revista brasileira de terapia comportamental e cognitiva, São Paulo, v. 10, n. 1, p. 105-118, 2008.

PACHECO, J.; ALVARENGA, P.; REPPOLD, C. ; PICCININI, C. A. ; HUTZ, C. S. Estabilidade do comportamento anti-social na transição da infância para a adolescência: uma perspectiva desenvolvimentista. Psicologia: reflexão e crítica, Porto Alegre, v. 18, n. 1, p. 55-62, 2005.

PACHECO, J.; SILVEIRA, L. M. O. B; SCHNEIDER, A. M. A. Estilos e práticas educativas parentais: análise da relação desses construtos sob a perspectiva do adolescente. Psico, Porto Alegre, v. 39, n. 1, p. 66-73, 2008. 
PATTERSON, G.; REID, J.; DISHION, T. Antisocial boys: comportamento anti-social. Santo André: Esetec, 2002.

PESCE, R. P.; ASSIS, S. G.; SANTOS, N.; OLIVEIRA, R. V. C. Risco e proteção: um equilíbrio promotor de resiliência. Psicologia: teoria e pesquisa, Brasília, v. 20, n. 2, p. 135-143, 2004.

PINHEIRO, M. I. S. ; HAASE, V. G; DEL PRETTE, A. ; AMARANTE, C. L. D; DEL PRETTE, Z. A. P. Treinamento de habilidades sociais educativas para pais de crianças com problemas de comportamento. Psicologia: reflexão e crítica, Porto Alegre, v. 19, n. 3, p. 407414, 2006.

REICHERT, C. B.; WAGNER, A. Autonomia na adolescência e sua relação com os estilos parentais. Psico, Porto Alegre, v. 38, n. 3, p. 292-299, 2007.

ROMER, D.; BLACK, M.; RICARDO, I. Social influences on the sexual behavior of youth at risk for HIV exposure. Journal public health, Oxford, v. 84, n.6, p. 977-985, 1994.

SALVO, C. G.; SILVARES, E. F. M.; TONI, P. M. Práticas educativas como forma de predição de problemas de comportamento e competência social. Estudos de psicologia, Natal, v. 22, n. 2, p. 187-195, 2005.

SCHENKER, M.; MINAYO, C. S. Fatores de risco e de proteção para o uso de drogas na adolescência. Ciência e saúde coletiva, Rio de Janeiro, v. 10, n. 3, p. 707-717, 2005.

SKINNER, B. F. Ciência e comportamento Humano. 10.ed. São Paulo: Martins Fontes, 1998.

STATTIN, H.; KERR, M. Parental Monitoring: A Reinterpretation. Child development, Raleigh, v. 71, n. 4, p. 1072-1085, 2000.

TEIXEIRA, M.; OLIVEIRA, A. M. ; WOTTRICH, S. H. Escalas de práticas parentais (EPP): avaliando dimensões de práticas parentais em relação a adolescentes. Psicologia: reflexão e crítica, Porto Alegre,v. 19, n. 3, p. 433-441, 2006.

TEIXEIRA, C. M. F. S. Vivência com pais de adolescentes: uma proposta de curso que facilita o relacionamento. Revista latino americana de enfermagem, Ribeirão Preto, v. 4, n. 2, p. 73-85, 1996.

WEBSTER-STRATTON, C.; REID, M. J.; HAMMOND, M. Preventing conduct problems, promoting social competence: $A$ parent and teacher training partnership in Head Start. J ournal of Clinical Child Psychology, New Orleans, v. 30, n. 3, p. 283-302, 2003.

\section{Endereço para correspondência}

Gabriela Mello Sabbag

Rua Schiller, no. 143, ap. 2001, CEP 80050-260,Curitiba - PR, Brasil

Endereço eletrônico: gabi_s@hotmail.com

Alessandra Turini Bolsoni-Silva

Rua Ana Rosa Zuicker D 'Annunziata, 2-27, CEP 17030-310, Bauru - SP, Brasil

Endereço eletrônico: bolsoni@fc.unesp.br 
Recebido em: 06/07/2010

Reformulado em: 10/09/2010

Aceito para publicação em: 13/09/2010

Acompanhamento do processo editorial: Adriana Benevides Soares

\section{Notas}

* Mestre em Psicologia do Desenvolvimento e Aprendizagem pela UNESP; Docente do curso de Psicologia da Faculdade Administração, Ciências, Educação e LetrasFACEL - Curitiba, PR.

**Pós-Doutorado em Saúde Mental, Bolsista Produtividade CNPq; Docente do curso de Psicologia e do Programa de Pós- graduação em Psicologia do Desenvolvimento e Aprendizagem da Faculdade de Ciências da Universidade do Estado de São Paulo - UNESP- Bauru, SP.

${ }^{1}$ Este trabalho é parte de um trabalho maior que culminou na Dissertação de Mestrado intitulada Análise das práticas e das habilidades sociais educativas maternas na interação com os filhos adolescentes da primeira autora com orientação da segunda. Contou com apoio da CAPES/CNPq.

${ }^{2}$ Os resultados apresentados indicam a necessidade de intervir na relação entre mães e filhos. Dos adolescentes, 144 perceberam que as mães faziam uso de práticas de risco, o que representa $52 \%$ da população que respondeu ao inventário de estilo materno. Somente 24 adolescentes, no entanto, perceberam que suas mães utilizavam práticas positivas, isto é, somente $9 \%$ tiveram uma percepção mais positiva sobre as práticas maternas. 